\title{
Polarimetric Synthetic Aperture Radar Data and the Complex Wishart Distribution
}

\author{
Allan Aasbjerg Nielsen ${ }^{1}$, Knut Conradsen ${ }^{1}$, and Henning Skriver ${ }^{2}$ \\ 1 IMM, Informatics and Mathematical Modelling, Building 321 \\ Technical University of Denmark, DK-2800 Kgs. Lyngby, Denmark

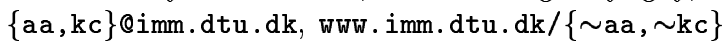 \\ 2 EMI, Section for Electromagnetic Systems, Ørsted $\bullet$ DTU, Building 348 \\ Technical University of Denmark, DK-2800 Kgs. Lyngby, Denmark \\ hs@oersted.dtu.dk, www.oersted.dtu.dk/staff/all/hs
}

\begin{abstract}
When working with multi-look fully polarimetric synthetic aperture radar (SAR) data an appropriate way of representing the backscattered signal consists of the so-called covariance matrix. For each pixel this is a $3 \times 3$ Hermitian, positive definite matrix which follows a complex Wishart distribution. Based on this distribution a test statistic for equality of two such matrices and an associated asymptotic probability for obtaining a smaller value of the test statistic are given and applied to segmentation, change detection and edge detection in polarimetric SAR data. In a case study EMISAR $L$-band data from 17 April 1998 and 20 May 1998 covering agricultural fields near Foulum, Denmark, are used.
\end{abstract}

\section{Introduction}

Due to its all-weather mapping capability and independence of, for instance, cloud cover, synthetic aperture radar (SAR) data hold a strong potential for e.g. segmentation, change detection and edge detection in remote sensing applications. It is well-known that different targets in different locations in a SAR image exhibit different backscatter characteristics. It is also well-known that the development of different targets (e.g. crops) over time causes changes in the backscatter. In the crop case the radar backscattering is sensitive to the dielectric properties of the vegetation and the soil, to the plant structure (i.e., the size, shape, and orientation distributions of the scatterers), to the surface roughness, and to the canopy structure (e.g. row direction and spacing, and cover fraction) $[1,2]$.

The polarimetric SAR measures the amplitude and phase of backscattered signals in four combinations of the linear receive and transmit polarizations: $\mathrm{HH}$, $\mathrm{HV}, \mathrm{VH}$, and $\mathrm{VV}$ ( $\mathrm{H}$ for horizontal and $\mathrm{V}$ for vertical polarization, respectively). These signals form the complex scattering matrix which relates the scattered to the incident electric fields [3]. The inherent speckle in the SAR data can be reduced by spatial averaging at the expense of loss of spatial resolution. In this so-called multi-look case a more appropriate representation of the backscattered signal is the covariance matrix in which the average properties of a group of 
resolution cells can be expressed in a single matrix. The average covariance matrix is defined as [3]

$$
\langle\boldsymbol{C}\rangle=\left[\begin{array}{ccc}
\left\langle S_{h h} S_{h h}^{*}\right\rangle & \left\langle S_{h h} S_{h v}^{*}\right\rangle & \left\langle S_{h h} S_{v v}^{*}\right\rangle \\
\left\langle S_{h v} S_{h h}^{*}\right\rangle & \left\langle S_{h v} S_{h v}^{*}\right\rangle & \left\langle S_{h v} S_{v v}^{*}\right\rangle \\
\left\langle S_{v v} S_{h h}^{*}\right\rangle & \left\langle S_{v v} S_{h v}^{*}\right\rangle & \left\langle S_{v v} S_{v v}^{*}\right\rangle
\end{array}\right]
$$

where $\langle\cdot\rangle$ denotes ensemble averaging, ${ }^{*}$ denotes complex conjugation, and $S_{r t}$ is the complex scattering amplitude for receive polarization $r$ and transmit polarization $t$ ( $r$ and $t$ are either $h$ for horizontal or $v$ for vertical). Reciprocity, which normally applies to natural targets, gives $S_{h v}=S_{v h}$ (in the backscattering direction using the backscattering alignment convention [3]) and results in the covariance matrix (1) with rank 3 . $\langle\boldsymbol{C}\rangle$ follows a complex Wishart distribution. The elements in the covariance matrix containing co- and cross-polarized components often contain little information. For randomly distributed targets with azimuthal symmetry the elements are zero [4].

In this paper a test statistic for equality of two complex covariance matrices and an associated asymptotic probability measure for obtaining a smaller value of the test statistic are given and applied to segmentation, change detection and edge detection in fully polarimetric SAR data. As opposed to existing methods that work on each polarization $(\mathrm{HH}, \mathrm{VV}, \mathrm{HV})$ individually followed by some heuristic fusion step, this new test statistic allows for the full polarimetric information simultaneously.

For a more thorough description of the method, see [5]; see also [6,7]. For the edge detection application, see [8-10]. For the segmentation application, see $[10,11]$.

\section{Theoretical Results}

This section gives the main results from the analysis carried out in [5] on the likelihood ratio test for equality of two complex Wishart matrices. For a more thorough description, see [5] and references therein.

The desired likelihood ratio test statistic for equality of two complex Wishart matrices is

$$
Q=\frac{(n+m)^{p(n+m)}}{n^{p n} m^{p m}} \frac{|\boldsymbol{X}|^{n}|\boldsymbol{Y}|^{m}}{|\boldsymbol{X}+\boldsymbol{Y}|^{n+m}}
$$

where $|\cdot|$ denotes the determinant. $n$ and $m$ are the number of looks, and $p=3$ is the dimension of the covariance matrix. For $n=m$ we get

$$
\ln Q=n(2 p \ln 2+\ln |\boldsymbol{X}|+\ln |\boldsymbol{Y}|-2 \ln |\boldsymbol{X}+\boldsymbol{Y}|) .
$$

The probability of finding a smaller value of $-2 \rho \ln Q$ is

$$
\begin{aligned}
& P\{-2 \rho \ln Q \leq z\} \simeq P\left\{\chi^{2}\left(p^{2}\right) \leq z\right\}+ \\
& \omega_{2}\left[P\left\{\chi^{2}\left(p^{2}+4\right) \leq z\right\}-P\left\{\chi^{2}\left(p^{2}\right) \leq z\right\}\right]
\end{aligned}
$$


where $\chi^{2}(f)$ denotes the chi-squared distribution with $f$ degrees of freedom,

$$
\rho=1-\frac{2 p^{2}-1}{6 p}\left(\frac{1}{n}+\frac{1}{m}-\frac{1}{n+m}\right)
$$

and

$$
\omega_{2}=-\frac{p^{2}}{4}\left(1-\frac{1}{\rho}\right)^{2}+\frac{p^{2}\left(p^{2}-1\right)}{24 \rho^{2}}\left(\frac{1}{n^{2}}+\frac{1}{m^{2}}-\frac{1}{(n+m)^{2}}\right) .
$$

For covariance matrix data $p=3$. For HH, HV or VV data $p=1$. In the latter case $\boldsymbol{X}$ and $\boldsymbol{Y}$ are therefore scalars $X$ and $Y$, and $Q$ reduces to

$$
Q=\frac{(n+m)^{n+m}}{n^{n} m^{m}} \frac{X^{n} Y^{m}}{(X+Y)^{n+m}}
$$

which is equivalent to the well-known likelihood ratio test statistic for the equality of two gamma parameters $[12,13]$.

The important case with azimuthal symmetry is not dealt with here. The elements in the covariance matrix containing co- and cross-polarized components often contain little information, and for randomly distributed targets with azimuthal symmetry these elements are zero. It is important to notice, however, that if the sample covariance matrix is forced into azimuthal symmetry, i.e., if the elements containing co- and cross-polarized components in the sample covariance matrix are set to zero, then the sample covariance matrix no longer follows the complex Wishart distribution. In [5] the test statistic and the asymptotic distribution are derived for the full covariance matrix case, for the azimuthal symmetric case, as well as for the diagonal case, where all off-diagonal elements are set equal to zero.

\section{Case Study: EMISAR Data}

The EMISAR is the result of a research and development project initiated in 1986 at the Department of Electromagnetic Systems (now Ørsted•DTU Department) of the Technical University of Denmark $[14,15]$. The EMISAR system is fully polarimetric and interferometric and it operates at two frequencies, $L$-band $(1.25 \mathrm{GHz} / 25 \mathrm{~cm}$ wavelength) and $C$-band $(5.3 \mathrm{GHz} / 5.7 \mathrm{~cm}$ wavelength). The EMISAR is flown on a Royal Danish Airforce Gulfstream G-3 aircraft and is normally operated from an altitude of approximately $12,500 \mathrm{~m}$, the spatial resolution is $2 \times 2 \mathrm{~m}^{2}$ (one-look), the ground range swath is approximately $12 \mathrm{~km}$, and typical incidence angles range from $35^{\circ}$ to $60^{\circ}$. The processed data from this system are fully calibrated by means of an advanced internal calibration system.

To illustrate the segmentation, change detection and edge detection capabilities of the derived test statistic this case study uses EMISAR data from an agricultural test site at the Research Center Foulum located in Central Jutland, Denmark. The data used here are part of the data used in a previously reported study on polarimetric signatures of crops [2]. 
Two EMISAR $L$-band images from 17 April 1998 and 20 May 1998 are shown in Figure 1 as color composites of the HV (actually the complex addition of $\mathrm{HV}$ and VH; red), HH (green), and VV (blue) channels. The HH and VV channels are stretched linearly from $-30 \mathrm{~dB}$ to $0 \mathrm{~dB}$ and the $\mathrm{HV}$ channel is strethed from $-36 \mathrm{~dB}$ to $-6 \mathrm{~dB}$. The single look scattering matrix data have been converted to covariance matrix data, and at the same time speckle reduced by a raised cosine filter to an equivalent number of looks of approximately $11(=n=m)$.

The geometrical co-registration is very important in a change detection application where two images are compared on a pixel-by-pixel basis. The polarimetric images were registered to a digital elevation model generated from interferometric data acquired by EMISAR. The registration was carried out by combining a model of the imaging geometry with a few ground control points, and the images were registered to one another with an rms-accuracy of better than one pixel [16].

Figure $3(\mathrm{a})$ shows the sum of the diagonal elements (actually $\mathrm{HH}+2 \mathrm{HV}+\mathrm{VV}$ ) in the covariance matrix data for the data acquired on 20 May 1998. This is the so-called span image.

Fields A and B are winter wheat and spring barley, respectively. The winter wheat field has a relatively high phase difference between $\mathrm{HH}$ and VV, probably because of double bounce scattering between the soil surface and the vegetation. The spring barley field has a low phase difference. Fields C and D are oats and peas fields, respectively. The oats field has a high and the peas field has a low phase difference.

\subsection{Segmentation}

The MUM (Merge Using Moments) segmentation algorithm for single-channel SAR images was proposed in [17]. As a first step the MUM algorithm severely over-segments the SAR image by dividing it into quadratic segments of size $k \times k$ pixels, where the value of $k$ depends on, e.g., the desired resolution in the resulting segmentation map and on the computational load. Below we use $k=2$. These initial, small segments are then merged based in their statistical properties. The MUM algorithm tries to determine appropriate regions in the image through an iterative region growing process of merging and sorting the segments. The sorting is performed because the order of the merging of the segments affects the final result. It should be noted that the merging performed in [17] is based on the Student's $t$-test, which is appropriate for Gaussian data, hence an improved segmentation result may be obtained by applying the proper test statistic for Gamma distributed intensities for single-channel SAR images as shown in [18]. Here, our new test statistic outlined above is used for segment merging in the multi-look polarimetric data.

Figure 2(a) shows segmentation using the diagonal elements in the $L$-band EMISAR data from 20 May 1998. Figure 2(b) shows segmentation using the azimuthal symmetric data from the same date. The colours chosen for the segments are arbitrary. 


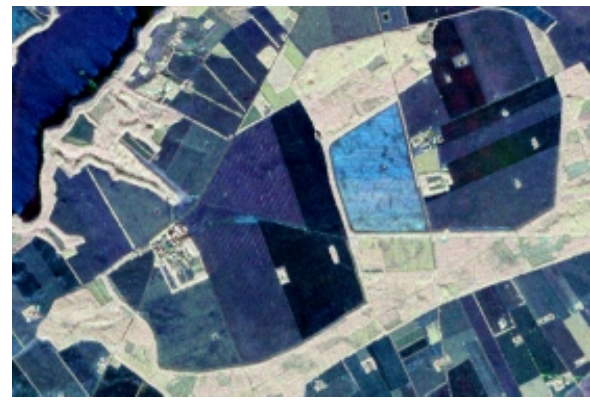

(a) 17 April 1998

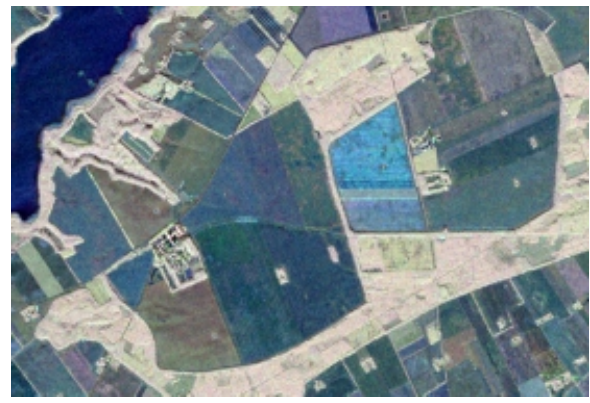

(b) 20 May 1998

Fig. 1. $L$-band EMISAR data

The advantage of including the polarimetric information in the segmentation algorithm is clearly seen. Especially, the difference is seen for the segments covering fields $\mathrm{A}$ and $\mathrm{B}$, and $\mathrm{C}$ and $\mathrm{D}$ (Figure 3(a)). For both cases, the two neighboring fields (i.e., $\mathrm{A}$ and $\mathrm{B}$, and $\mathrm{C}$ and $\mathrm{D}$, respectively) are merged when only the backscatter coefficients are used (Figure 2(a)), whereas they are not merged when the polarimetric information is included (Figure 2(b)).

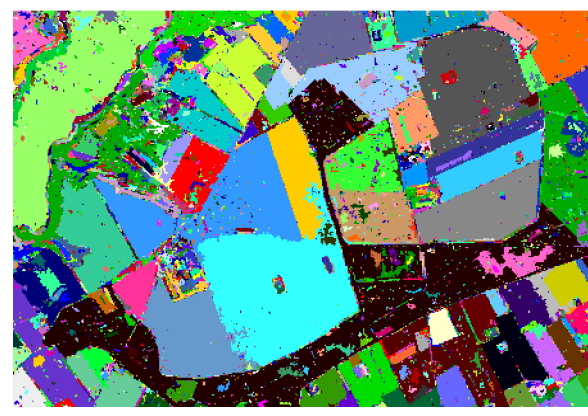

(a) Segmentation using diagonal elements

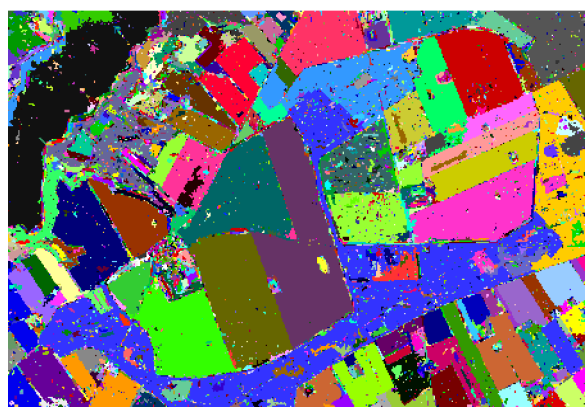

(b) Segmentation using azimuthal symmetry

Fig. 2. $L$-band EMISAR data from 20 May 1998

\subsection{Change Detection}

Figure 3(b) shows $\ln Q$ for the full covariance matrix data stretched linearly between -50 and 0 . Where $Q$ is close to 1 and therefore $\ln Q$ is close to 0 which are the bright regions no or little change occurred between the two points in time. Where $\ln Q$ is small which are the dark regions change did occur.

The large bright areas in Figure 1 are mostly coniferous forests and we observe very small change for these areas (Figure 3(b)) due to the very stable 


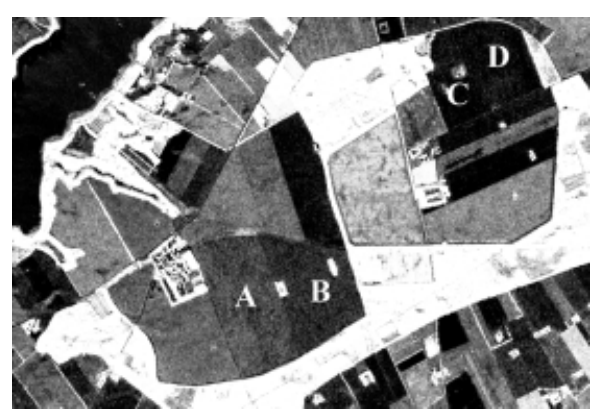

(a) Span image

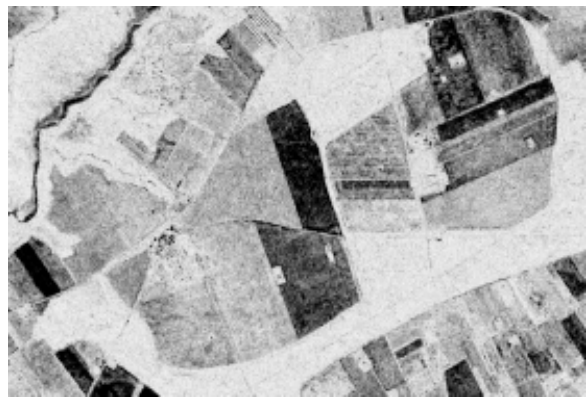

(b) Change image, $\ln Q$, full covariance matrix

Fig. 3. $L$-band EMISAR data from 20 May 1998

backscatter from such forest areas (see e.g. [19]). The agricultural fields show different degrees of change, dependent on the crop type. To the extreme left in the image between the lake in the upper left and the coniferous forest three small fields show change, no-change and change behavior, respectively. The field with no change is a beet field, which for both acquisitions is a bare field. The two other fields are spring barley fields, which are bare at the first acquisition and have a $12-14 \mathrm{~cm}$ vegetation layer at the second acquisition. The three large fields (marked B and A in Figure 3(a) and the field to the immediate left of field A) in the image are from left to right: grass, winter wheat (A) and spring barley (B). The grass field is virtually unchanged between the two acquisitions. The vegetation height for the winter wheat field (A) has changed from 14 to $42 \mathrm{~cm}$ between the two acquisitions, and we observe a moderate change between the images. It is obvious from the results presented here and the more detailed evaluation in [5] that the new change detection method is working very well when changes in the polarimetric parameters are experienced.

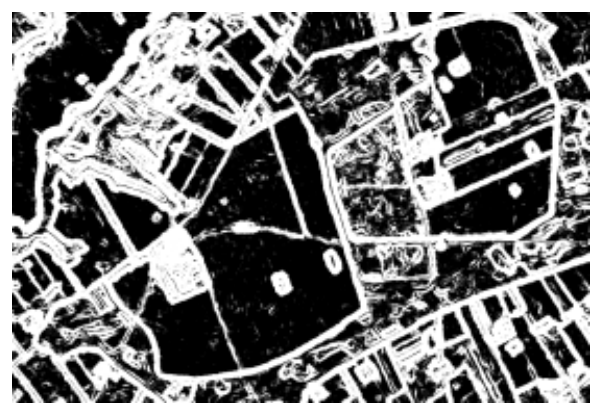

(a) Edges using diagonal elements

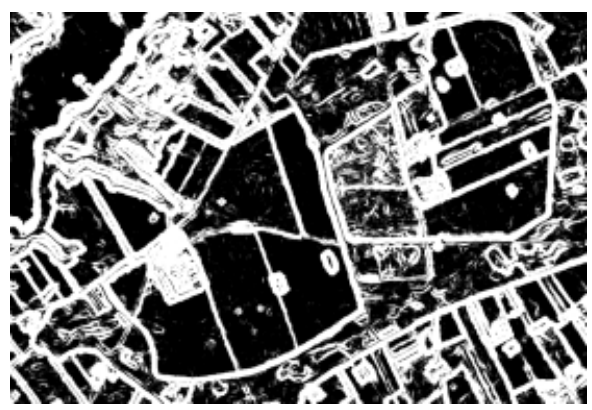

(b) Edges using azimuthal symmetry

Fig. 4. $L$-band EMISAR data from 20 May 1998 


\subsection{Edge Detection}

The edge detection is performed by visiting each pixel in the image in succession, and for each pixel applying a set of filters with different orientations. The filters estimate the mean covariance matrices on each side of the central pixel, and the test of equality of these two mean covariance matrices determines whether an edge is detected by the current filter or not; see $[8,9]$ for further details.

Figure 4(a) shows edges detected using the diagonal elements in the $L$-band EMISAR data from 20 May 1998. Figure 4(b) shows edges detected using the azimuthal symmetric data from the same date.

The advantage of including the polarimetric information in the edge detection is clearly seen. Especially, the difference is seen for the edges between fields $\mathrm{A}$ and $\mathrm{B}$, and $\mathrm{C}$ and $\mathrm{D}$, respectively (Figure 3(a)). In these cases, no edge is detected when only the backscatter coefficients are used in the edge detection (Figure 4(a)), whereas the edge is detected when the polarimetric information is included (Figure $4(\mathrm{~b})$ ).

\section{Conclusion}

A new test statistic for the equality of two matrices following the complex Wishart distribution is given with an asymptotic probability for obtaining a smaller value of the test statistic. These measures are applied to segmentation, change detection and edge detection in polarimetric synthetic aperture radar data. The new test statistic and the associated probability measure combines the full polarimetric signal in a unified way. As opposed to existing methods that deal with each polarization marginally in turn followed by some heuristic fusion step, this new test statistic allows for the full polarimetric information simultaneously.

\section{References}

1. F. T. Ulaby, R. K. Moore, and A. K. Fung, Microwave Remote Sensing: Active and Passive, Artech, Dedham, MA, 1986, vol. 3.

2. H. Skriver, M. T. Svendsen, and A. G. Thomsen, "Multitemporal $L$ - and $C$-band polarimetric signatures of crops," IEEE Transactions on Geoscience and Remote Sensing, vol. 37, pp. 2413-2429, 1999.

3. J. J. van Zyl and F. T. Ulaby, "Scattering matrix representation for simple targets," in Radar Polarimetry for Geoscience Applications, F. T. Ulaby and C. Elachi, Eds. Artech, Norwood, MA, 1990.

4. S. V. Nghiem, S. H. Yueh, R. Kwok, and F. K. Li, "Symmetry properties in polarimetric remote sensing," Radio Science, vol. 27, no. 5, pp. 693-711, 1992.

5. K. Conradsen, A. A. Nielsen, J. Schou, and H. Skriver, "A test statistic in the complex Wishart distribution and its application to change detection in polarimetric SAR data," Accepted for IEEE Transactions on Geoscience and Remote Sensing, 2002. 
6. K. Conradsen, A. A. Nielsen, H. Skriver, and J. Schou, "Change detection in polarimetric SAR data and the complex Wishart distribution," in Proceedings of the International Geoscience and Remote Sensing Symposium (IGARSS), Sydney, New South Wales, Australia, 9-13 July 2001.

7. H. Skriver, A. A. Nielsen, and K. Conradsen, "Evaluation of the Wishart test statistic for polarimetric data," Invited to the International Geoscience and Remote Sensing Symposium (IGARSS), Toulouse, France, 21-25 July 2003.

8. J. Schou, H. Skriver, K. Conradsen, and A. A. Nielsen, "CFAR edge detector for polarimetric SAR data," Accepted for IEEE Transactions on Geoscience and Remote Sensing, 2002.

9. H. Skriver, J. Schou, K. Conradsen, and A. A. Nielsen, "Polarimetric edge detector based on the complex Wishart distribution," in Proceedings of the International Geoscience and Remote Sensing Symposium (IGARSS), Sydney, New South Wales, Australia, 9-13 July 2001.

10. J. Schou, Feature Extraction for Topographic Mapping, Ph.D. thesis, Ørsted•DTU, Technical University of Denmark, Lyngby, 2001.

11. H. Skriver, J. Schou, A. A. Nielsen, and K. Conradsen, "Polarimetric segmentation using the complex Wishart test statistic," in Proceedings of the International Geoscience and Remote Sensing Symposium (IGARSS), Toronto, Ontario, Canada, 24-28 June 2002.

12. R. Touzi, A. Lopes, and P. Bousquez, "A statistical and geometrical edge detector for SAR images," IEEE Transactions on Geoscience and Remote Sensing, vol. 26, no. 6 , pp. $764-773$, Nov. 1988.

13. A. Lopes, E. Nezry, R. Touzi, and H. Laur, "Structure detection and statistical adaptive speckle filtering in SAR images," International Journal of Remote Sensing, vol. 13, no. 9, pp. 1735-1758, 1993.

14. S. N. Madsen, E. L. Christensen, N. Skou, and J. Dall, "The Danish SAR system: Design and initial tests," IEEE Transactions on Geoscience and Remote Sensing, vol. 29 , pp. $417-476,1991$.

15. E. L. Christensen, N. Skou, J. Dall, K. Woelders, J. H. Jørgensen, J. Granholm, and S. N. Madsen, "EMISAR: An absolutely calibrated polarimetric $L$ - and $C$ band SAR," IEEE Transactions on Geoscience and Remote Sensing, vol. 36, pp. 1852-1865, 1998.

16. W. Dierking, J. Schou, and H. Skriver, "Change detection of small objects and linear features in multi-temporal polarimetric images," in Proceedings of the International Geoscience and Remote Sensing Symposium (IGARSS), IEEE, Ed., Honolulu, Hawaii, USA, 24-28 July 2000, pp. 1693-1695.

17. R. Cook, I. McConnell, and C. Oliver, "MUM (Merging Using Moments) segmentation for SAR images," in SPIE vol. 2316, 1994, pp. 92-103.

18. C. Oliver and S. Quegan, Understanding Synthetic Aperture Radar Images, Artech House, 1998.

19. W. Dierking and H. Skriver, "Change detection for thematic mapping by means of airborne multi-temporal polarimetric SAR imagery," IEEE Transactions on Geoscience and Remote Sensing, vol. 40, no. 3, pp. 618-636, 2002. 